\title{
Emotion-aware Computing using Smartphone
}

\author{
Surjya Ghosh \\ Department of Computer Science and Engineering \\ Indian Institute of Technology Kharagpur, India \\ surjya.ghosh@iitkgp.ac.in
}

\section{INTRODUCTION}

In this project, we address the problem to determine human emotion states automatically using modern day smartphones. Sensor-rich smartphones have opened up the opportunity to unobtrusively collect user behaviour patterns, activity details and infer information about emotion states. Determining human emotion accurately and efficiently to build a scalable system is the major objective of the project. Towards that goal, we plan to develop an emotion detection model leveraging on different information sources present on smartphone.

Emotion awareness is finding its applicability in various applications in digital marketing, healthcare, advertising, personalized learning, and in designing novel user interfaces [1]. We discuss two specific scenarios Quantified self ${ }^{1}$ and Affective learning [8], where this can be useful.

\section{Research Objective}

We outline the various subproblems towards the broad objective of designing and implementing a non-intrusive, accurate and efficient smartphone based emotion detection system.

\section{A. Adaptive Sampling}

Today's smartphone contains myriad information sources like call log, voice data, app usage pattern, browsing history, SMS, email log, which carry personal footprints and can be correlated with human emotion. However, sampling data from multiple sources is challenging as it makes the monitoring process complex and resource-intensive. Also, as usage pattern of one channel is different from another, it requires a large amount of data collection for extracting features from each source, which essentially increases the training period to build the emotion detection model.

We want to build an adaptive sampling mechanism, which can explore different information sources for the user based on her usage pattern and sample usage details from the targeted channel at right interval in an energy efficient manner.

\section{B. Identify Ideal Information Source}

Apart from monitoring overhead, resource overhead, prolonged data collection period of multiple information sources, there are other challenges in terms of interpretability if multiple information sources are used to infer emotion. In case of multiple channels, often the relationship between a particular channel and inferred emotion is not well understood. Usage

\footnotetext{
${ }^{1}$ http://quantifiedself.com/.
}

of a single information source can alleviate this problem and make the system simple, interpretable and energy-efficient.

So, we plan to explore if there is any 'ideal' information source that can be used for emotion detection. We believe exploration of typing activity in smartphone can help in this regard since the usage of different typing applications is on the rise [6] and people often express their emotion using different instant messaging applications. Moreover, for desktop PCs, it has been shown that emotion states can be identified from keyboard dynamics [2]. Apart from this typing being a noninvasive and energy-efficient modality, it can overcome the problem of energy.

\section{Combine Multiple Information}

Adaptive Sampling can help to explore multiple information sources. It is important to realize that one activity channel (e.g. call history) is intrinsically different from another (e.g. Online Social Network activity). So, we categorize every source channel with the set of parameters - influence / weight vector of the activity. Identifying and selecting appropriate parameters for a channel that can impact the accuracy are major tasks. In case of multi-channel strategies the task becomes even harder to identify the right combinations of parameters from different channels to attain a very high level of accuracy.

\section{Energy Constraints and Privacy}

Once the user emotion can be predicted accurately, we want to ensure that the energy constraints are honoured and the privacy of the user is not compromised. Since every information channel has different energy-constraints, an important research objective is to perform an 'energy-accuracy' tradeoff to optimally combine the activity channels to get accurate prediction. Similarly, selecting appropriate channels based on user allowed privacy discloser level to enure emotion prediction quality is also an important research question.

\section{E. Applications}

We believe the developed smartphone based emotion detection application and the exposed interface can be useful in multiple scenarios. In context Quantified self this can be helpful. It can track emotion variations of the user and also provide emotion-aware recommendations. Similarly in the area of Affective learning, this application can identify user frustration or confusion and accordingly can change the difficulty level of the course. 


\section{RELATED WORK}

We present relevant works that use smartphone as a device for collecting data for emotion detection.

\section{A. Smartphone-based Emotion Recognition Techniques}

A number of works exploit the smartphone usage details and build a multi-state emotion prediction model. MoodScope proposed to infer mood exploiting multiple information channels, such as SMS, email, phone call patterns, application usage, web browsing, and location [7]. In EmotionSense, Rachuri et al. used multiple features from the Emotional Prosody Speech and Transcripts library to train the emotion classifier [10]. Similarly, Pielot et al. tried to infer boredom from smartphone usage pattern like call details, sensor details, etc., and used 35 features [9].

\section{B. Touch-based Emotion Recognition Techniques}

Widespread availability of touch-based devices and steady increase [6] in the usage of instant messaging apps open a new possibility of inferring emotion from touch interactions. For example, Lee et al. designed a Twitter client app and collected data from various on-board sensors including typing to predict emotion [5]. Similarly, Gao et al. used multiple finger-stroke related features to identify different emotional states during a touch based game play [3]. Ciman et al. detected stress conditions by analyzing multiple features of swipe, scroll and text input interactions in a smartphone [11].

On the contrary, we select an energy-efficient, non-intrusive information source i.e. typing in smartphone and implement a smartphone-based emotion detection system.

\section{PRoposed APPROACH}

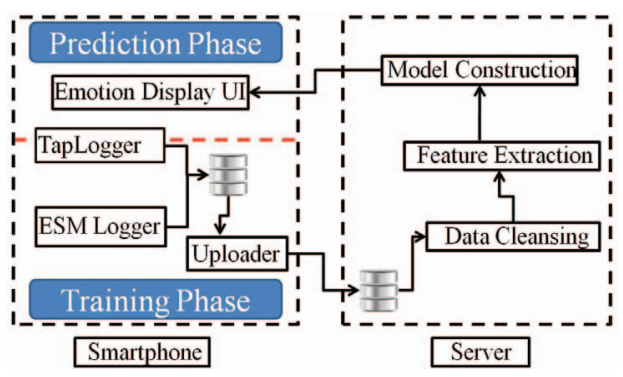

Fig. 1: TapSense architecture

We have started initial explorations towards the broad project objective of designing an efficient and accurate smartphone based emotion detection system. We have designed and implemented an Android based prototype application TapSense (Figure 1), to trace user's typing activity in smartphone and record her emotion states. We have asked users to provide emotion labels (such as happy, sad, stressed and relaxed), which are used as emotion ground truth. We have also identified typing sessions as users perform typing and extracted different keystroke related features - typing speed, number of backspace or delete keys, number of special characters, session text length, session duration, last recorded emotion from every session. Later these features are correlated with the emotion label to using a Random Forest based personalized, multi-state emotion detection model.

\section{PREliminary Result}
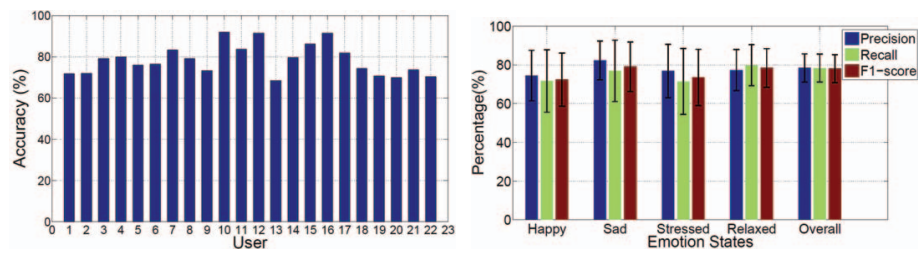

(a) Accuracy of predicting different

(b) Precision, Recall and F1-score emotions across all users

Fig. 2: Performance evaluation of classification model

We have evaluated the prototype using with a 3 -week uncontrolled study involving 22 participants. It shows that we can identify multiple emotion states with an average accuracy of $78 \%$ (Figure 2a). We also observe that individual states are also identified with an average precision ranging from $74 \%$ to $82 \%$ (Figure $2 \mathrm{~b}$ ). We have also shown that a carefully designed emotion ground truth approach [4] can outperform the existing approaches in terms of user engagement and accuracy of emotion classification.

\section{FUTURE WORK}

We shall continue the explorations towards the broad objective of implementing a privacy preserving and energy efficient emotion detection system using multiple information sources present on smartphone and plan to design emotion aware recommendations in context of quantified self and affective learning.

\section{REFERENCES}

[1] R. Calvo, S. D'Mello, et al. Frontiers of affect-aware learning technologies. Intelligent Systems, IEEE, 27(6):86-89, 2012.

[2] C. Epp, M. Lippold, and R. L. Mandryk. Identifying emotional states using keystroke dynamics. In ACM SIGCHI, pages 715-724, 2011.

[3] Y. Gao, N. Bianchi-Berthouze, and H. Meng. What does touch tell us about emotions in touchscreen-based gameplay? ACM Trans. on Computer Human Interactions, 19(4), Dec. 2012.

[4] S. Ghosh, N. Ganguly, B. Mitra, and P. De. Towards designing an intelligent experience sampling method for emotion detection. In IEEE CCNC, Accepted, 2017.

[5] H. Lee, Y. S. Choi, S. Lee, and I. Park. Towards unobtrusive emotion recognition for affective social communication. In IEEE CCNC, 2012.

[6] U. Lee, J. Lee, M. Ko, C. Lee, Y. Kim, S. Yang, K. Yatani, G. Gweon, K.-M. Chung, and J. Song. Hooked on smartphones: an exploratory study on smartphone overuse among college students. In ACM SIGCHI, pages 2327-2336, 2014.

[7] R. LiKamWa, Y. Liu, N. D. Lane, and L. Zhong. Moodscope: Building a mood sensor from smartphone usage patterns. In ACM Mobisys, 2013.

[8] R. W. Picard, S. Papert, W. Bender, B. Blumberg, C. Breazeal, D. Cavallo, T. Machover, M. Resnick, D. Roy, and C. Strohecker. Affective learninga manifesto. BT technology journal, 22(4):253-269, 2004.

[9] M. Pielot, T. Dingler, J. S. Pedro, and N. Oliver. When attention is not scarce-detecting boredom from mobile phone usage. In ACM UbiComp. pages 825-836, 2015.

[10] K. K. Rachuri, M. Musolesi, C. Mascolo, P. J. Rentfrow, C. Longworth, and A. Aucinas. Emotionsense: A mobile phones based adaptive platform for experimental social psychology research. In ACM UbiComp, 2010.

[11] K. Wac, M. Ciman, and O. Gaggi. isensestress: Assessing stress through human-smartphone interaction analysis. In IEEE PervasiveHealth, pages 8-p, 2015. 